\title{
Relationship between Periodontitis and Diabetes - Importance of a Clinical Study to Prove the Vicious Cycle
}

\author{
Toshiyuki Nagasawa ${ }^{1}$, Mitsuhiko Noda ${ }^{2}$, Sayaka Katagiri ${ }^{3}$, Maki Takaichi ${ }^{2}$, \\ Yoshihiko Takahashi ${ }^{2}$, Nawarat Wara-Aswapati ${ }^{4}$, Hiroaki Kobayashi ${ }^{3}$, Satoko Ohara ${ }^{5}$, \\ Yoko Kawaguchi ${ }^{6}$, Tadashi Tagami ${ }^{7}$, Yasushi Furuichi ${ }^{1}$ and Yuichi Izumi ${ }^{3,8}$
}

\begin{abstract}
Periodontitis is regarded to have a close relationship to diabetes mellitus. In Japan, some cohort studies have indicated that there is a significant positive association of obesity plus metabolic syndrome with periodontal diseases. As such, an increasing number of studies suggest a relationship between periodontitis and diabetes, most of which are epidemiologic association. In this review, we have summarized the possible evidence of a relationship between periodontitis and diabetes. To date, little evidence has been reported to indicate that diabetes and/or glucose intolerance has in fact had a significant cause-effect relationship with periodontal disease. In this regard, it is important to directly uncover the relation, i.e., to prove the effect of therapeutic approaches to periodontitis upon mitigation of glycemic control in diabetic patients, which would be a direct evidence of its causal nature. Therefore a study should be undertaken to this effect.
\end{abstract}

Key words: periodontitis, diabetes

(Inter Med 49: 881-885, 2010)

(DOI: 10.2169/internalmedicine.49.3351)

\section{Introduction}

Periodontitis is a chronic inflammatory disease characterized by periodontal pocket formation, loss of connective tissue attachment and alveolar bone resorption, which can eventually result in tooth loss. When oral hygiene is compromised, oral bacteria form a plaque biofilm, which is resistant to chemicals and immune cells. Without mechanical debridement, the plaque biofilm matures and causes gingivitis within a few days. Gingivitis represents chronic but reversible inflammation and can be treated by proper plaque control. Gingivitis usually does not extend to irreversible periodontitis for months or years. Recent investigations have raised the possibility that periodontitis and obesity are re- lated to each other, and this might cause vicious cycle of diabetes and periodontitis (Fig. 1).

In this context, periodontitis is regarded as having a close relationship to diabetes mellitus (1) in many ethnic groups. In Japan, some cohort studies indicate that there is a significant positive association of both upper body obesity (2) and metabolic syndrome (3) with periodontal diseases. However, to date, there is no direct epidemiologic evidence in a large population that diabetes and/or glucose intolerance may have a significant positive relationship to periodontal disease in Japanese subjects. In fact, one study i.e., the Hisayama study, a currently ongoing longitudinal population-based observational study, found positive relationships of body mass index (BMI) and waist-hip ratio to periodontal disease whereas neither impaired glucose tolerance nor diabetes was

\footnotetext{
${ }^{1}$ Division of Periodontology and Endodontology, School of Dentistry, Health Science University of Hokkaido, Hokkaido, ${ }^{2}$ Department of Diabetes and Metabolic Medicine, National Center for Global Health and Medicine, Tokyo, ${ }^{3}$ Section of Periodontology, Department of Hard Tissue Engineering, Graduate School, Tokyo Medical and Dental University, Tokyo, ${ }^{4}$ Department of Periodontology, Faculty of Dentistry, Khon Kaen University, Khon Kaen, Thailand, ${ }^{5}$ Oral Diagnosis and General Dentistry, Dental Hospital, Faculty of Dentistry, Tokyo Medical and Dental University, Tokyo, ${ }^{6}$ Department of Oral Health Promotion, Graduate School, Tokyo Medical and Dental University, Tokyo, ${ }^{7}$ Department of Dentistry and Oral Surgery, National Center for Global Health and Medicine, Tokyo and ${ }^{8}$ Global Center for Excellence for Tooth and Bone Research, Tokyo Medical and Dental University, Tokyo
}

Received for publication January 6, 2010; Accepted for publication January 28, 2010

Correspondence to Dr. Mitsuhiko Noda, mnoda@ hosp.ncgm.go.jp 


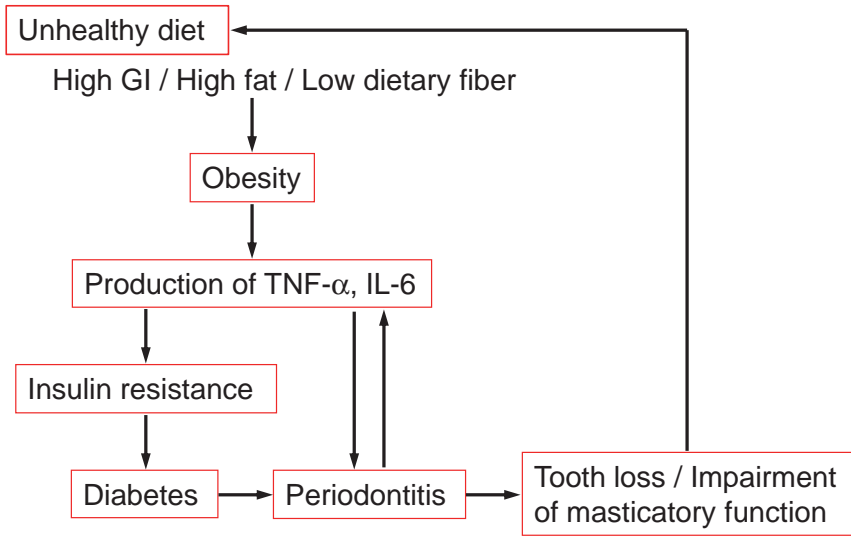

Figure 1. Vicious spiral of periodontitis and diabetes. Recent research on the association between periodontitis and diabetes and/or obesity may imply that periodontitis and obesity are related to each other, and that this relationship might result in a vicious cycle.

significantly associated with probing pocket depth (4). Moreover, no convincing evidence has yet been obtained regarding whether therapeutic approaches to periodontal disease can in fact mitigate glycemic control in diabetic patients with or without antibiotic treatment (5-7), although several studies are currently underway (8).

Increasing evidence suggests the relationship between periodontitis and diabetes. In this review, we have summarized the evidence of the relationship between periodontitis and diabetes, in the following sections.

\section{Possible Relationship of Diabetes to Periodontitis - Epidemiologic View}

Loe reported that periodontitis is the 6th complication of diabetes (9). Among 2,273 Pima Indians, the age- and gender-adjusted prevalence of periodontal disease was $60 \%$ in type 2 diabetic patients and $36 \%$ in those without diabetes at the first examination (10). In a subset of 701 subjects who initially had little or no evidence of periodontal disease, periodontitis newly developed in 22 patients. The rate of periodontal disease in subjects with diabetes was 2.6 times (5\% CI 1.0-6.6, controlled for age and gender) greater than that in those without diabetes (10). The prevalence of periodontitis in Japanese was significantly higher in both type 1 and type 2 diabetic patients than in non-diabetic subjects (11). These results suggest that type 1 and type 2 diabetes are both risk factors for periodontitis. The high prevalence of periodontitis among diabetic patients is thought to be mainly due to their high susceptibility to infection (12).

\section{Possible Relationship of Periodontitis to Diabetes}

Taylor et al followed glycemic control in type 1 diabetic patients for 2 years, and reported that those with periodontitis had an increased risk for worsening glycemic con- trol (13). In addition, patients with periodontitis had increased risks of ketoacidosis, retinopathy and neuropathy as compared with diabetic patients who did not have periodontitis. Diabetic patients with neurological complications had significantly more severe gingivitis than those without this complication (14).

Inflammatory cytokines including TNF- $\alpha$ and IL-6 are thought to induce insulin resistance. Stimulation of fat cells with TNF- $\alpha$ phosphorylates insulin-receptor substrate- 1 and impairs insulin receptor tyrosine kinase (15). Uysal et al reported that mice lacking TNF- $\alpha$ function were resistant to obesity-induced insulin resistance (16). Administration of IL-6 to healthy volunteers induced dose-dependent increases in fasting blood glucose (17). These results suggest that the inflammatory cytokines, which induce insulin resistance and chronic inflammatory diseases including periodontitis, might augment insulin resistance through the production of inflammatory cytokines within the lesion itself. In addition, both TNF- $\alpha$ and IL- 6 are produced in adipose tissue, and onethird of circulating IL- 6 is also derived from adipose tissue (18). These lines of evidence suggest that obesity, diabetes and periodontitis are mutually related to each other.

\section{Association between Periodontitis and Diabetes through Obesity}

Obesity is caused by high calorie and high fat diets, which are well known causes of type 2 diabetes. Control of diet is important for the treatment of diabetes.

The glycemic index (GI) is an index used to determine the physiological basis for carbohydrate absorption. In general, foods that require adequate mastication have low GI and are considered to be healthy. In the context of a healthy diet, preservation of masticatory function is important for control of blood glucose levels. Nowjack-Raymer and Sheiham examined the dental status and intake of nutritious foods in a US civilian non-institutionalized population (NHANES III). It was reported that the intake of carrots and tossed salads among denture-wearers were 2.1 and 1.5 times lower than for the fully dentate, respectively, and dietary fiber intake was 1.2 times lower (19). The observation that people with fewer than 28 teeth had significantly lower intakes of carrots, tossed salads, and dietary fiber than did fully dentate people, suggested dental status to significantly affect both diet and nutrition. The NHANES III study found that subjects with diabetes and insulin resistance had more severe periodontitis than those without these conditions (20). A model was proposed in which obesity, caused by excessive food consumption, induced TNF- $\alpha$ production from fat tissue, resulting in insulin resistance, diabetes and periodontitis (20). As edentulous subjects were apt to consume sweet snacks compared to those who still had teeth (21), edentulism might impair healthy food intake, leading to obesity. Periodontitis is the major cause of tooth loss in the adult population, and advanced periodontitis impairs masticatory function, suggesting that periodontitis might impact diabetes 


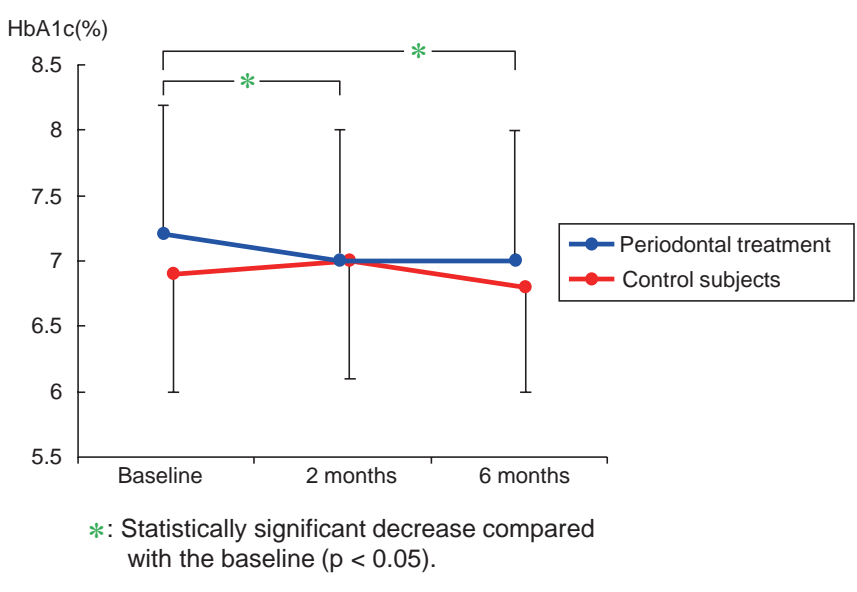

Figure 2. Effect of periodontal treatment on HbA1c in type 2 diabetic patients. Periodontal treatment with topical antibiotics improved HbA1c through reduction of hs-CRP, which may relate to amelioration of insulin resistance in type 2 diabetic patients with periodontal disease (25). *: Statistically significant decrease compared with the baseline $(\mathrm{p}<0.05)$.

via the impairment of appropriate nutritional intake.

\section{Effects of Periodontal Treatment on Diabetes}

Several studies showed that periodontal treatment significantly improved glycemic conditions (22-25). A recent meta-analysis of the efficacy of periodontal treatment on glycemic control in diabetic patients suggested that such treatment could lead to significant reductions in HbA1c (26). However, they also suggested that the results need to be viewed with caution due to a lack of robustness and limitations in the designs of some of the studies included (26).

We have examined the effects of periodontal treatment with topical antibiotics on HbA1c levels and serum highsensitivity C-reactive protein (hs-CRP) values in type 2 diabetic patients with periodontal disease (27). Periodontal treatment in the test group $(n=32)$ transiently reduced HbA1c levels without changing hs-CRP, while the control group $(n=17)$ showed no changes in either HbA1c or hsCRP. Multiple regression analysis of all subjects revealed that BMI and changes in hs-CRP levels correlated significantly with HbA1c reduction at 6 months after periodontal treatment. Based on the results of multiple regression analysis, the intervention group was divided into two subgroups: those in which hs-CRP levels decreased (CRP-D group), and those in which hs-CRP levels were unchanged or increased (CRP-N group) ( $\mathrm{n}=16$, each). Re-analysis was conducted based on these subgroups. In the CRP-D subgroup, HbA1c was significantly reduced at the end of the study, but did not decrease in the CRP-N subgroup. The HbA1c decrease in the CRP-D subgroup following periodontal treatment was significantly greater than that in the CRP-N subgroup. The BMI of each group remained unchanged at the end of the study. Thus, the results suggested that periodontal treatment with topical antibiotics improves HbAlc by reducing hsCRP, which may relate to amelioration of insulin resistance in type 2 diabetic patients with periodontal disease (27) (Fig. 2).

\section{How to Test the Hypothesis}

Although we have summarized above the possible evidence of a relationship between periodontitis and diabetes, much of the evidence is epidemiologic evidence and no convincing evidence has yet been obtained regarding whether therapeutic approaches to periodontal disease can in fact have a beneficial effect on glycemic control in diabetic patients, with or without antibiotic treatment (5-7), although several studies are currently underway (8). In this regard, it is important to directly uncover the relation, i.e., to prove the effect of therapeutic approaches to periodontitis upon glycemic levels of diabetic patients, which would be a direct evidence of its causal nature.

We therefore are planning a study (TMDU-IMCJ Periodontitis Study) to elucidate whether treatment of periodontal disease may have such a glucose-lowering effect in Japanese type 2 diabetic patients, as will be described below. This study would be expected to yield direct evidence of the effects of periodontal treatment in the Japanese population. Our trial is a 24-week follow-up observation with periodontal intervention including antibiotic treatment.

\section{TMDU-IMCJ Periodontitis Study}

Objectives: The main objective of this study will be to assess whether periodontal treatment leads to a decrease in HbA1c levels in type 2 diabetic patients with periodontitis.

Study participants: Subjects are to be recruited in the Department of Diabetes and Metabolic Medicine, International Medical Center of Japan (IMCJ; currently, National Center for Global Health and Medicine), Tokyo, Japan. Subjects are to be selected in two steps: a screening visit (Visit 0 ; V0) at the department and an inclusion visit at the Department of Dentistry of IMCJ on the same day. Periodontal treatments and follow-up visits are performed at the Periodontology section of Tokyo Medical and Dental University (TMDU). These two institutes, IMCJ and TMDU, are within -30 minutes of each other by subway.

Eligibility, inclusion, and exclusion criteria: shown in Table 1.

Intervention: Subjects satisfying the inclusion criteria and exempt from the exclusion criteria at $\mathrm{V} 0$ will be given the chance to participate in the trial and will be given a scheduled appointment (V1) at the Section of Periodontology of TMDU within -4 weeks after V0 when they consent to take part in this study. Those who decline are followed up at the Department of Dentistry of IMCJ with obtainment of their consent. Periodontal treatment includes non-surgical scaling and root planing, topical antibiotic therapy, and oral health instructions. These are performed at V1-V5 (V1 and 1-4 
Table 1. Eligibility, Inclusion, and Exclusion Criteria of TMDU-IMCJ Periodontitis Study

\begin{tabular}{ll}
\hline Eligibility criteria: & periodontal probing depth (PPD) $\geq 4 \mathrm{~mm}$ \\
$\begin{array}{l}\text { During the study period, patients who consult } \\
\text { on at least } 4 \text { teeth. }\end{array}$ & $\begin{array}{l}\text { Exclusion criteria: } \\
\text { the Diabetes Department of IMCJ are screened }\end{array}$ \\
$\begin{array}{l}\text { for possible inclusion in the trial. } \\
\text { Inclusion criteria: }\end{array}$ & $\begin{array}{l}\text { months prior toV0 } \\
\text { - } \geq 40 \text { and }<80 \text { years old }\end{array}$ \\
- have type 2 diabetes & - taking non-steroidal anti-inflammatory \\
- HbA1c between 6.5 - $10.0 \%$ at V0) & - hypersensitivity to tetracyclines \\
- have had stable antidiabetic therapy during & - known infectious diseases \\
the two months preceding V0 & - under treatment for malignant disease(s) \\
- differences in HbA1c at V0 and previous & - have valvular disease(s) of the heart \\
values during this period $\leq 1 \%$ & - reduced number of platelets or coagulation \\
- have at least four natural permanent teeth & disorder(s) \\
- have periodontitis; periodontitis is defined & - taking anti-platelet drug(s) and/or warfarin. \\
by the presence of one site with a & \\
\hline
\end{tabular}

weeks after V1).

Outcome measures: HbAlc, glycoalbumin, CRP, white blood cell counts, HOMA-R, number of teeth having site(s) with $P P D \geq 4 \mathrm{~mm}$, and mean PPD.

\section{Conclusions and Future Perspectives}

Periodontitis and diabetes are related to each other, and this might produce a vicious cycle (Fig. 1). Treatment of periodontitis could potentially alleviate this downward spiral. Further intervention studies are however needed to determine the effect of periodontal treatment on the glycemic control in diabetic patients.

\section{References}

1. Katz PP, Wirthlin MR Jr, Szpunar SM, Selby JV, Sepe SJ, Showstack JA. Epidemiology and prevention of periodontal disease in individuals with diabetes. Diabetes Care 14: 375-385, 1991.

2. Saito T, Shimazaki Y, Koga T, Tsuzuki M, Ohshima A. Relationship between upper body obesity and periodontitis. J Dent Res 80: 1631-1636, 2001.

3. Shimazaki Y, Saito T, Yonemoto K, Kiyohara Y, Iida M, Yamashita Y. Relationship of metabolic syndrome to periodontal disease in Japanese women: The Hisayama Study. J Dent Res 86: 271-275, 2007.

4. Saito T, Shimazaki Y, Kiyohara Y, et al. Relationship between obesity, glucose tolerance, and periodontal disease in Japanese women: the Hisayama study. J Periodont Res 40: 346-353, 2005.

5. O'Connell PA, Taba M, Nomizo A, et al. Effects of periodontal therapy on glycemic control and inflammatory markers. J Periodontol 79: 774-783, 2008.

6. Llambés F, Silvestre FJ, Hernández-Mijares A, Guiha R, Caffesse $\mathrm{R}$. The effect of periodontal treatment on metabolic control of type 1 diabetes mellitus. Clin Oral Investig 12: 337-343, 2008.

7. Rodrigues DC, Taba MJ, Novaes AB, Souza SL, Grisi MF. Effect of non-surgical periodontal therapy on glycemic control in patients with type 2 diabetes mellitus. J Periodontol 74: 1361-1367, 2003.

8. Vergnes JN, Arrivé E, Gourdy P, et al. Periodontal treatment to improve glycaemic control in diabetic patients: study protocol of the randomized, controlled DIAPERIO trial. Trials 10: 65, 2009.

9. Loe H. Periodontal disease. The sixth complication of diabetes mellitus. Diabetes Care 16: 329-334, 1993.

10. Nelson RG, Shlossman M, Budding LM, et al. Periodontal disease and NIDDM in Pima Indians. Diabetes Care 13: 836-840, 1990.

11. Nishimura $F$, Kono $T$, Fujimoto $C$, Iwamoto $Y$, Murayama $Y$. Negative effects of chronic inflammatory periodontal disease on diabetes mellitus. J Int Acad Periodontol 2: 49-55, 2000.
12. Genco RJ. Current view of risk factors for periodontal diseases. J Periodontol 67: 1041-1049, 1996.

13. Taylor GW, Burt BA, Becker MP, et al. Severe periodontitis and risk for poor glycemic control in patients with non-insulindependent diabetes mellitus. J Periodontol 67 (Suppl): 1085-1093, 1996.

14. Rosenthal IM, Abrams H, Kopczyk A. The relationship of inflammatory periodontal disease to diabetic status in insulin-dependent diabetes mellitus patients. J Clin Periodontol 15: 425-429, 1988.

15. Hotamisligil GS, Peraldi $P$, Budavari A, Ellis R, White MF, Spiegelman BM. IRS-1-mediated inhibition of insulin receptor tyrosine kinase activity in TNF-alpha- and obesity-induced insulin resistance. Science 271: 665-668, 1996.

16. Uysal KT, Wiesbrock SM, Marino MW, Hotamisligil GS. Protection from obesity-induced insulin resistance in mice lacking TNFalpha function. Nature 389: 610-614, 1997.

17. Tsigos C, Papanicolaou DA, Kyrou I, Defensor R, Mitsiadis CS, Chrousos GP. Dose-dependent effects of recombinant human interleukin-6 on glucose regulation. J Clin Endocrinol Metab 82: 4167-4170, 1997.

18. Mohamed-Ali V, Goodrick S, Rawesh A, et al. Subcutaneous adipose tissue releases interleukin-6, but not tumor necrosis factoralpha, in vivo. J Clin Endocrinol Metab 82: 4196-4200, 1997.

19. Nowjack-Raymer RE, Sheiham A. Association of edentulism and diet and nutrition in US adults. J Dent Res 82: 123-126, 2003.

20. Genco RJ, Grossi SG, Ho A, Nishimura F, Murayama Y. A proposed model linking inflammation to obesity, diabetes, and periodontal infections. J Periodontol 76 (Suppl): 2075-2084, 2005.

21. Johansson I, Tidehag P, Lundberg V, Hallmans G. Dental status, diet and cardiovascular risk factors in middle-aged people in northern Sweden. Community Dent Oral Epidemiol 22: 431-436, 1994. 
22. Rodrigues DC, Taba MJ, Novaes AB, Souza SL, Grisi MF. Effect of non-surgical periodontal therapy on glycemic control in patients with type 2 diabetes mellitus. J Periodontol 74: 1361-1367, 2003.

23. Grossi SG, Skrepcinski FB, DeCaro T, et al. Treatment of periodontal disease in diabetics reduces glycated hemoglobin. J Periodontol 68: 713-719, 1997.

24. Kiran M, Arpak N, Unsal E, Erdoğan MF. The effect of improved periodontal health on metabolic control in type 2 diabetes mellitus. J Clin Periodontol 32: 266-272, 2005.

25. Jones JA, Miller DR, Wehler CJ, et al. Does periodontal care improve glycemic control? The Department of Veterans Affairs Dental Diabetes Study. J Clin Periodontol 34: 46-52, 2007.
26. Darré L, Vergnes JN, Gourdy P, Sixou M. Efficacy of periodontal treatment on glycaemic control in diabetic patients: A metaanalysis of interventional studies. Diabetes Metab 34: 497-506, 2008.

27. Katagiri $S$, Nitta $H$, Nagasawa $T$, et al. Multi-center intervention study on glycohemoglobin (HbA1c) and serum, high-sensitivity CRP (hs-CRP) after local anti-infectious periodontal treatment in type 2 diabetic patients with periodontal disease. Diabetes Res Clin Pract 83: 308-315, 2009.

(C) 2010 The Japanese Society of Internal Medicine http://www.naika.or.jp/imindex.html 